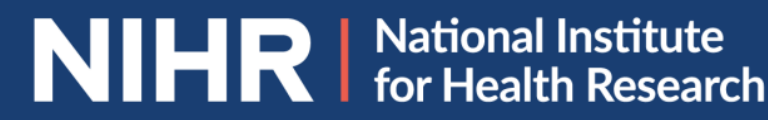

\title{
Living with Covid19
}

A dynamic review of the evidence around ongoing Covid19 symptoms (often called Long Covid)

Author: Dr Elaine Maxwell 
Contents

Key Messages 3

Introduction 4

What are we aiming to achieve with our Living with Covid19 review? 4

Drawing on people's experience 4

How many people live with ongoing Covid19?

Symptoms 6

Diagnostic uncertainty $\quad 8$

Research into living with Covid19 9

Capturing the experience of 'Long Covid' 10

Services needed $\quad 15$

Resources 16

Commissioned research $\quad 20$

Early conclusions $\quad 22$

Next steps $\quad 23$ 


\section{Key Messages}

The novel coronavirus (Covid19) pandemic declared by the World Health Organisation in March 2020 has had far-reaching effects upon people's lives, health care systems and wider society. As yet there is little research into the number of people at risk of developing ongoing Covid19. Early attention has been on the acute illness generated by the virus, but it is becoming clear that, for some people, Covid19 infection is a long term illness.

This rapid and dynamic review draws on the lived experience of patients and expert consensus as well as published evidence to better understand the impact of ongoing effects of Covid19, how health and social care services should respond, and what future research questions might be. Our steering group concluded:

- There is a widespread perception that people either die, get admitted to hospital or recover after two weeks. It is increasingly clear that for some people there is a distinct pathway of ongoing effects. There is an urgent need to better understand the symptom journey and the clinical risks that underlie that. People, their families and healthcare professionals need realistic expectations about what to expect.

- A major obstacle is the lack of consensus on diagnostic criteria for ongoing Covid19. A working diagnosis that is recognised by healthcare services, employers and government agencies would facilitate access to much needed support and provide the basis for planning appropriate services. Whilst it is too early to give a precise definition, guidance on reaching a working diagnosis and a code for clinical datasets is needed.

- The fluctuating and multi-system symptoms need to be acknowledged. A common theme is that symptoms arise in one physiological system then abate only for symptoms to arise in a different system.

- There are significant psychological and social impacts that will have long term consequences for individuals and for society if not well managed

- The multisystem nature of ongoing Covid19 means that it needs to be considered holistically (both in service provision and in research). The varying degrees of dependency mean support in the community should be considered alongside hospital one stop clinics. Social support needs to be understood together with the financial pressures on previously economically active people.

- Covid19 has a disproportionate effect on certain parts of the population, including care home residents. Black and Asian communities have seen high death rates and there are concerns about other minority groups and the socially disadvantaged. These people are already seldom heard in research as well as travellers, the homeless, those in prisons, people with mental health problems or learning difficulties; each having particular and distinct needs in relation to ongoing Covid19 that need to be understood. 


\section{Introduction}

The National Institute for Health Research Centre for Engagement and Dissemination (NIHR CED) aims to engage people in knowledge exchange in order to develop and improve health and social care. One of the ways we do this is through our Themed Reviews. These are not systematic reviews of all the evidence. Nor are they guidance or recommendations. Instead they are narratives based on a selection of different kinds of evidence chosen to describe the current state of knowledge and to inform discussions, focused on actions for practice. Themed Reviews include both academic study and practical wisdom from lived experience and are guided by a steering group of practitioners, researchers and public members.

\section{What are we aiming to achieve with our Living with Covid19 review?}

This review differs from our previous reviews. Given the importance of the subject matter and the need for information to ensure people receive care now, we have taken the unusual decision to write this review despite the relatively small amount of published evidence. We worked with a steering group and a patient reference group who provided us with a broad range of expertise and perspectives. Their insight helped deepen our understanding of the new phenomenon 'ongoing Covid19' in people who do not recover after a short period of illness

New evidence is emerging daily. Given the lack of definition of the condition, assimilating evidence into a systematic review is difficult. Our steering group believe a more useful contribution will be to frame different types of evidence with questions about how to set it in context. Our steering group felt it is important to retain rather than synthesise different perspectives (epidemiologist, researcher, policy makers, professionals and patients) when considering evidence, noting and learning when there are mismatches in assumptions. Exploring these different viewpoints also reveals gaps in current commissioned research.

We hope this review will be useful to the public, health and social care professionals, researchers, service providers and policy makers and lead to better understanding of the issues around living with Covid19. Given work underway by NICE and others in developing comprehensive guidelines using the best available evidence, we do not seek to discuss clinical interventions in detail, although we point people to useful expert consensus resources.

By posting evidence now, we are encouraging a dynamic cycle of feedback and refinement. We want all readers, whatever their background, to work with us to develop our collective understanding and help improve care for people living with Covid19. This first iteration is necessarily far from comprehensive and will contain many gaps and omissions which we hope readers will help us remedy by providing information, links to resources and research studies that we have yet to tap.

At various points we provide feedback boxes and encourage readers to share their evidence, experience and thoughts. We will add resources to the site as they become 
available and will publish a substantive review of the emerging evidence in February 2021.

\section{Drawing on people's experience}

There is, as yet, limited and incomplete evidence on the long-term effects of Covid19 partly because it has not been possible to fully research them this early in the pandemic. However, it is becoming increasingly clear that, for some people, Covid19 infection is not a discrete episode but one that marks the start of ongoing and often debilitating symptoms. For some, this is related to their rehabilitation following a hospital admission, but others are reporting life-changing experiences that follow an initial infection that they managed at home, with symptoms becoming more severe over time.

Around the world, people with experience of living with ongoing effects of Covid19 are collating the range of experience and sharing their coping strategies, but as yet there is no formal diagnostic term for these ongoing effects, nor services to support them. For many people, social media is their main source of support.

"I sobbed when I found a Long Covid Facebook group ... I then found I wasn't alone" Nikki

People experiencing similar symptoms call it "Long Covid" or "Long Haul Covid" but it is unclear if all of the people are suffering from the same phenomenon. Many researchers and healthcare professionals are therefore cautious about attributing all the reported problems to a single diagnosis. In the light of this concern we have opted to use the phrases 'ongoing Covid19' and 'living with Covid19' until evidence can support either one or more specific diagnostic definitions. However, the lack of a single diagnostic category in no way diminishes the very real and often severe continuing impact living with Covid19 has on people's lives. Treatment and support are needed now and are necessarily based on expert consensus and lived experience as well as emergent research findings.

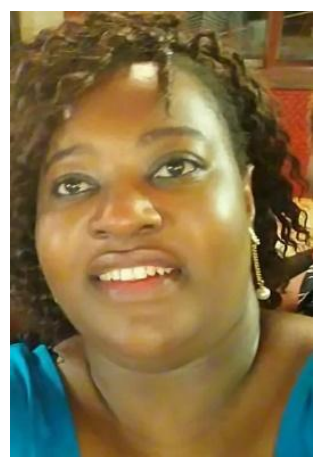

Early April, two days after testing positive I was hospitalised due to Covid infection with bilateral pneumonia where I spent 11 most terrifying days. My symptoms included breathlessness, uncontrolled fever, raging sore throat and vomiting. I felt as though life was being sucked out every minute and I thought I would die. Once, I saw 'the light', but something pulled me away. My faith was tested but the thoughts of my family, prayers and reassurance from hospital staff to fight this dreadful virus kept me going. Looking back, it was as if I was watching a film but feeling the pain and anguish. On ICU, I was attached to a breathing machine called a CPAP hood and needing assistance with almost everything. However, I eventually made it back home and I thought recovery would be much easier compared to the nightmare I had already endured except it was the beginning of a different kind of a nightmare - 'Long Covid'. Although I see this as silent, it is the quite debilitating with symptoms of severe fatigue, recurrent sore throats and brain fog. All this has had an immense impact not only on 
my health but family, social and work life and what makes it even worse is no-one knows how long I am likely to take to fully recover. Pretty

\section{How many people live with ongoing Covid19?}

Covid19 began to emerge at the end of 2019 and as yet there is little research into the number of people at risk of developing ongoing Covid19 or the duration of their symptoms. An unreferenced but frequently cited estimate is that most people recover from 'mild' infections within two weeks and more serious disease within three weeks.

We are at an early stage of understanding the disease and need to be careful not to draw conclusions prematurely. Even in a discrete occupational group such as people serving in the defence services, the incidence is unclear with new referrals continuing to the military Covid19 rehabilitation service from people who were first unwell in March 2020.

However, a number of small surveys are reporting remarkably similar findings which challenge that assumption. A team from Italy, one of the earliest affected countries, reported that $87 \%$ of people discharged from a Rome hospital were still experiencing at least one symptom 60 days after the onset on Covid 19 and $55 \%$ had three or more symptoms including fatigue (53\%), difficulty in breathing (43\%), joint pain (27\%), and chest pain (22\%) with $40 \%$ saying it had reduced the quality of their life ${ }^{1}$. On 5 th June 2020, NHS England published 'After-care needs of inpatients recovering from COVID19'. This estimated that up to that date, more than 95,000 patients had been admitted to hospitals across England with Covid 19 and it assumed $45 \%$ would need ongoing support ${ }^{2}$. Some estimates suggest that up to $50 \%$ of people hospitalised would need formal rehabilitation services ${ }^{3}$.

An unknown number of people have had Covid19 symptoms (both with and without access to a test) and self-cared at home. Public Health England published guidance on 7 th September 2020 stating that around $10 \%$ of 'mild' Covid19 cases who were not admitted to hospital have reported symptoms lasting more than four weeks and a number of hospitalised cases reported continuing symptoms for eight or more weeks following discharge 4 .

Similar findings are reported by a patient group who surveyed a self-selected sample of 640 people with symptoms lasting over two weeks (respondents were predominantly US based white females and not hospitalised at any time). The vast majority of participants experienced fluctuations in the type of symptoms $(70 \%)$ and the intensity of symptoms (89\%) over time ${ }^{5}$. The UK Covid Symptom Study app 6 has over 4 million regular contributors and suggests that a 'significant number' of people report symptoms for a month and between $10 \%$ and $20 \%$ report complications for longer.

Both people who were admitted to hospital and those who remained at home are reporting persistent chronic problems, including fatigue, myalgia and anxiety, with varying degrees of intensity. The Royal College of $\mathrm{GPs}^{7}$ anticipates a significant workload related to the ongoing health consequences related to respiratory difficulties 
and mental health problems such as anxiety, depression, adjustment disorder and post-traumatic stress disorder (PTSD).

It is becoming clear that there is a significant health and social care need that is likely to grow as more people are exposed to Covid19. This need is likely to become a permanent demand on health and social care services, even after the pandemic subsides.

\section{Symptoms}

"COVID-19 is clearly a complex multisystem disease and not just restricted to the cardiopulmonary systems. This is so, both in the acute phase and the post-acute recovery phase. Our service personnel who are undergoing multidisciplinary inpatient rehabilitation, at DMRC Stanford Hall, for their post COVID-19 sequalae commonly complain of fatigue (which can be severe), myalgias, anxiety, low mood, cognitive problems and atypical chest pain."

Group Captain Alex Bennett, Defence Professor of Rheumatology and Rehabilitation, Stanford Hall

Whilst the range of symptoms and their relative frequency is not fully documented, it is clear that Covid19 can affect not only the respiratory system but also the heart and cardiovascular system, the brain directly (encephalitis) and indirectly (e.g., secondary to low levels of oxygen or blood clots), the kidneys and the gut ${ }^{8}$. In August 2020, a NICE rapid review reported that acute myocardial injury is the most commonly described cardiovascular complication in Covid19, occurring in $8-12 \%$ of all those discharged with heart failure and arrythmias ${ }^{9}$. Ongoing problems with the liver and skin have also been reported.

Evidence from previous corona virus outbreaks indicates the risk of ongoing virusrelated effects. Approximately $30 \%$ of survivors of global SARS and MERS outbreaks experienced persistent physiological impairment and abnormal radiology consistent with fibrotic lung disease ${ }^{10.11}$ as well as long term heart, lung, blood glucose, brain and psychological complications ${ }^{12}$. A prospective cohort study of people with SARS found up to a third had persistent lung damage ${ }^{13}$.

"Five days after lockdown I woke during the night and thought "Oh! l've got a sore throat...... hope it's not the virus." The sore throat persisted through the following days along with an upset stomach and feeling overheated after any physical activity, although my temperature read as elevated but not "fever".

Five days after my symptoms started my husband announced that he had lost his taste and smell. "Well, that's it then" I thought, "must be the virus." No tests were available to confirm.

From week four I started to get chest pains and then breathlessness, gradually other symptoms developed including dry mouth, sore tongue, joint pains, fatigue, rash and tachycardia.

The following weeks were frightening as symptoms fluctuated; sometimes thinking that you were improving and then very disheartening when they returned. It wasn't until 
mid-June when I found the Long Covid Facebook group that I realised I wasn't alone, thousands of people were in the same situation. Knowing this helped enormously. After nearly 6 months I have started to feel some improvement, although doing anything remotely physical results in a flare up of symptoms. I couldn't have survived this without the love and support of my husband and family." Julia

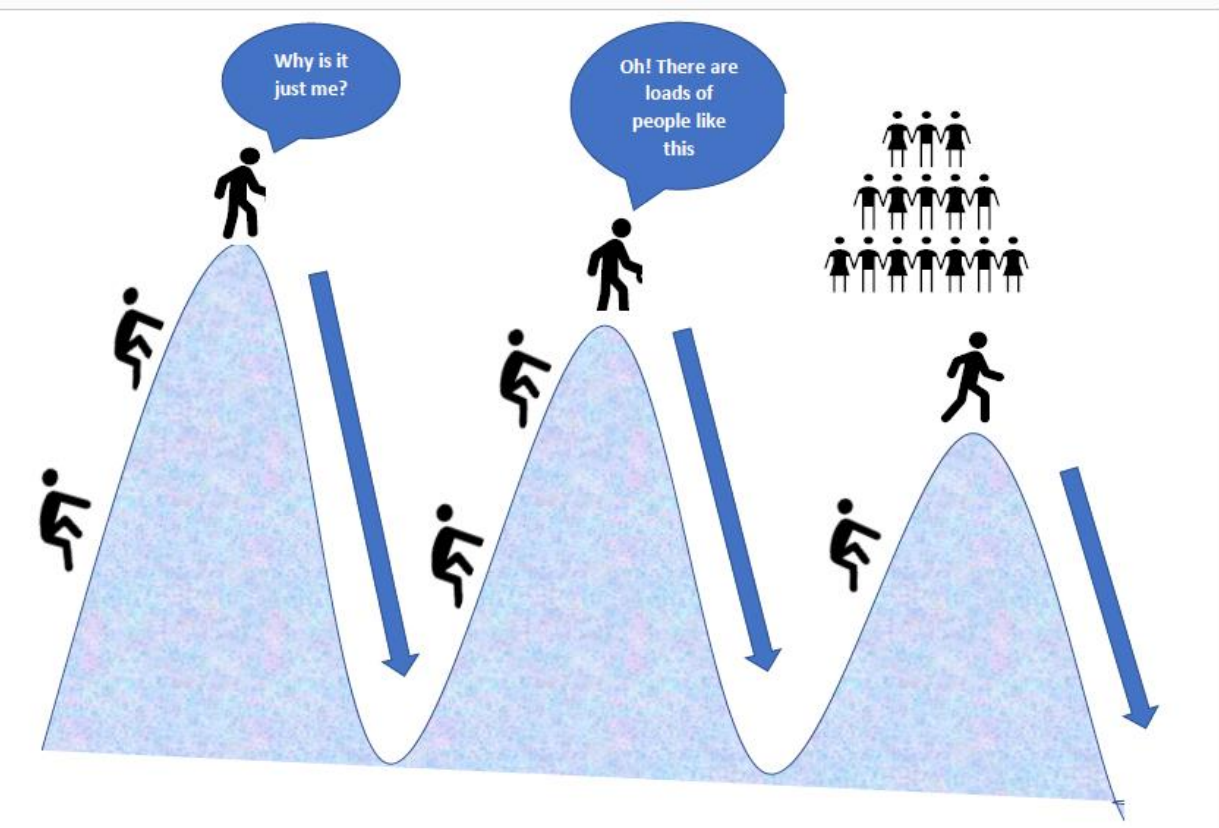

Previous coronavirus infections have also been associated with high levels of emotional distress. A Canadian study of 117 people with SARS found $17 \%$ had not returned to work 1 year later and $44 \%$ had used mental health services ${ }^{14}$. A systematic review of the mental health of people with SARS concluded that in the early stages of recovery there is a fear for survival and fear of infecting others. Later concerns are around perceived stigmatization, reduced quality of life, and psychological/emotional distress. Post-traumatic stress symptoms were found in high proportions of survivors even as late as 51 months after the initial infection infection ${ }^{15}$.

Members of the steering group also felt it is important to note that some of the mental health issues may relate to 'lockdown' conditions rather than to the virus itself. There is a well-established literature on the impact of living with a long-term condition on mental health, and of the complex interactions between physical and psychological symptoms in so-called 'medically unexplained symptoms'. An important area for research will be the interactions between the two, recognising mental health issues as both a possible consequence of living with Covid19 and as a potential consequence of having ongoing physical symptoms.

"the symptoms cause anxiety not vice versa" Jo

We are looking for more evidence on the nature and duration of ongoing symptoms. If you know of any studies we'd missed, please let us know here 


\section{Diagnostic uncertainty}

As yet there is no diagnostic code for 'Long Covid' meaning this experience is not captured in routine clinical datasets. 'Long Covid' is a term widely used on social media but is not a well-defined term and not a diagnosis used widely by clinical staff. The lack of empirical diagnostic tests may mean that a number of different conditions may be falling under a single umbrella term. This does not mean the steering group feel that testing for the Covid19 virus is a prerequisite to being considered to be ill with ongoing Covid19. Indeed, some expressed reservation about relying on testing, having seen a number of patients who have had a series of negative tests but with blood count and chest $x$ ray clearly showing Covid19. It was noted that some people have drawn parallels with post-viral syndrome, although the general view of the group was that it is too early to conclude that the experience of living with Covid19 is always post-viral syndrome. Indeed there is a possibility that the symptoms described may be due to a number of different syndromes (e.g. Post Intensive Care syndrome, Post Viral Fatigue syndrome and Long Term Covid syndrome). Some people may be suffering with more than one syndrome at the same time.

Some members of the steering group cautioned against settling on a fixed definition at this early stage of understanding the disease and felt the International Classification of Functioning, Disability and Health (ICF-developed by the World Health Organization) provides a better standard language and a framework for the description of health and health-related state.

However, the consequence of not having a formal diagnosis also has implications. For example, the military Covid19 rehabilitation service found that the psychological impact of not having a formal Covid19 diagnosis can inhibit people from engaging with their rehabilitation programme.

For others, a diagnosis is a gateway to getting support and access to specialist services. Many people had their initial symptoms in April and May 2020 when community testing in England was suspended. Symptomatic people were unable to get tested and are left in limbo, without a positive test but past the stage at which they would test positive. For those who were not admitted to hospital, there is often no other clinical data and they are reliant on people believing their history of the disease.

The steering group felt it important to acknowledge the term used by people with experience of living with Covid19 without committing everyone to using the same term and to encourage people to be mindful not to put all ongoing symptoms down to Covid19, when these may be attributable to other concurrent diseases.

My covid journey began in February 2020 where I became unwell with a cold, fever and cough. At the time I didn't really notice I was unwell but over the following few weeks I noticed that I seemed to be unable to recover and I was now suffering with a crippling fatigue as well as the other symptoms of exhaustion, a strange taste and brain fog. Whereas before I was a relatively fit person and wouldn't think twice at 
walking over 5/6 miles on a daily basis I would now struggle to walk to the end of my street. I also found social situations really hard and couldn't keep up with conversations and online meetings were a real problem. Fortunately, as an opera singer I haven't lost the quality or power in my singing voice and it is in really good form. I currently feel I am living in a "Covid" cycle of symptoms (fever, cough and metallic taste), extreme fatigue and brain fog then a few days of normality. Slowly,

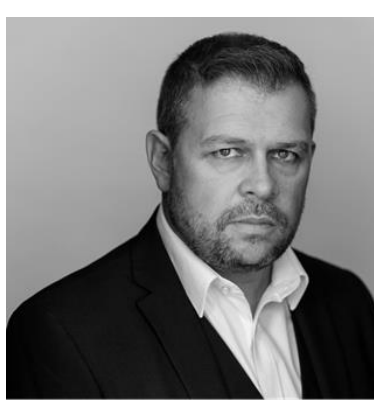
after nearly six months I am slowly beginning to see more 'normal' days but as soon as I begin to feel better the cycle starts again. I have found great support on the "Long Covid Support Group" on Facebook as there have been many moments where l've truly felt on my own although my family and wife have been incredibly supportive. I do feel I am now beginning to see the light at the end of this endless "Covid tunnel" and wish to offer support and hope to other sufferers out there. Lee

Research into living with Covid19

Research into public health control of the virus, critical care support and vaccine development commissioned by the NIHR and others has been conducted in record time and early findings have already changed the management of individual patients dramatically. More research is now required into ongoing needs, including psychological and social needs, together with research into policy and organisation of health and social care services.

The Steering Group recognised that service provision is mirrored by the fragmentation of research studies, with much of the commissioned research looking at a single symptom or physiological system rather than the whole experience, although there are notable exceptions such as the Defence Services/University of Oxford M-COVID study which is investigating the complications and outcomes of Covid19 infection in a young/middle aged physically active population (in this case, a military population).

Our steering group felt the challenge at this point in time is to design research that integrates reported lived experience needs with clinical models of care. The emerging scale of the challenge of living with Covid19 means this needs to be done rapidly. There is also a need for the social and psychological consequences of ongoing Covid19 to be considered as part of clinical care models. How to do this whilst still having an incomplete understanding of the range of symptoms, their links to specific pathologies, and optimal treatment is a difficult conundrum. It requires flexibility and openness to new information, wherever it comes from whilst also conducting rigorous, high quality research.

We have heard powerful stories that ongoing Covid19 symptoms are experienced by all age groups, from the youngest children to the oldest people, and people from all backgrounds. We cannot assume that groups who are at low risk of life threatening disease and death during acute infections are also at low risk on living with Covid19 long term. 
Acute Covid19 has disproportionately affected certain groups in society who are already underserved by research such as those with Learning Disabilities, mental health problems, older and frail people, and those with many other long term conditions as well as people from Black, Asian and Minority ethnicities. We do not know if they also have increased risk of ongoing symptoms. For example, we know that internationally $19-72 \%$ of Covid19 deaths occurred in care homes ${ }^{16}$, but it is unclear how many care home residents who survived the initial infection have ongoing Covid19. Research into the risk factors for living with Covid19 is an immediate priority in order to inform effective service planning.

\section{Capturing the experience of 'Long Covid'}

In the absence of detailed research into the lived experience, we held a focus group with some members of the Long Covid Facebook group (which currently has over 20,000 members) and agreed a summary report with them. Four overarching themes emerged from the discussion: expectations, symptom journey, being doubted, and support.

\section{Expectations}

\section{Key points}

Covid19 is not always a linear disease with an acute phase followed by recovery or a steady state rehabilitation. It can be cyclical disease, with symptoms moving round different body systems and fluctuating in severity

Our focus group acknowledged the initial difficulties in providing information about ongoing Covid19 but felt that official expectations had not been sufficiently updated with the learning since the pandemic was first confirmed. The group recognised that for many people, Covid19 is a relatively mild illness and they recover quickly:

"I have relatives, friends and colleagues who only experienced symptoms maximum of 2-3 weeks and are back to their usual self now" Pretty

There is growing evidence that the disease takes a different path for others, one that is not well documented and not yet well understood. The emphasis on acute respiratory problems creates an impression that anything else is a 'mild case' ignoring the devastating impact of other symptoms. A number of people said there were occasions when they thought they might not survive the night and there was anger at being labelled 'mild cases' simply because they didn't access respiratory support in hospitals. Using the term 'mild' for these people's disease was seen to diminish their experience and the group perceive that this has coloured the views of the both the public and of health professionals toward 'Long Covid':

"I think the term "mild" should be removed... I know that people who were admitted to the hospital were worse, but we who stayed home did not have MILD cases in all cases" Kelly 
In England, there was a strong message, reinforced by the call line NHS 111, that for most people not admitted to hospital, recovery would take around two weeks. People who had not been admitted to hospital expected to convalesce for around two weeks, citing the UK Government advice to phone NHS 111 if symptoms had not improved after seven days. Official advice that Covid19 presents with a limited number of symptoms (fever, cough and breathlessness - latterly updated to include change in sense of smell) meant some people with atypical presentations were not tested early, or in some cases not tested at all. All members of our focus group initially expected that there would be a short recovery period, including those who had been admitted to critical care.

The public perception of Covid19 was perceived to be in binary terms, as either severe enough to need hospital admission or mild enough to recover quickly and not to require support. The failure to acknowledge a third path, together with the media focus on mortality rates, created anxiety in those with continuing symptoms who were left in an uncertain no man's land about the severity of their condition:

"There was this, either you're going to end up in hospital and die or you're going to recover. My husband and I got it from helping a neighbour who died within five days of testing positive, so there's that fear and we made our wills and clung on in there" Jo

This perception, combined with the UK government message to stay away from health services unless very ill, left many people uncertain about when they should seek help. Many of the group were expecting flu like or upper respiratory tract infections and were surprised when they either had mild or absent respiratory problems. Those with long term conditions such as asthma who had previous experience of chest infections expected the recovery to follow their past experience and were perplexed that it did not. They were surprised when other symptoms emerged and only then sought help from health services. There was and still is little discussion of the other symptoms in official advice, leading people to social media sites to discuss and compare their experiences:

"I think the message to avoid hospital and the GP unless you had specific symptoms was very unhelpful, particularly as I didn't have, and never have had, a cough or fever" Edward

Focus group members who had been hospitalised expected to need more time to recover than those who stayed at home, based on their observations of other patients. One person who had received Continuous Positive Airway Pressure (CPAP) oxygen therapy assumed it would take three weeks to recover. She was dismayed that she is still unable to work months later. Another who had been on a ventilator for 18 days was initially told recovery would take one week for every day in Intensive Care Unit but has since had that estimate revised upwards a number of times.

The lack of clear and frequently updated information led to inconsistent advice from healthcare professionals. Our focus group said they would have preferred a wellinformed "we don't know". Specialist staff such as physiotherapists and medical staff working in dedicated Covid centres gave longer estimates than GPs, but even these were sometimes still inaccurate. 
"I finally had a respiratory appointment three months later, over the phone (not over a video link). I was told I am so unwell because l'm not moving enough and recommended graded exercise. When I then saw a rehabilitation physiotherapist, she said no, we are not going to do graded exercise because that would be counterproductive for you. How are we to know what is good for us and what is not when there is conflicting information?' Francesca

The consequences were significant as people tried to carry on despite not having recovered, rather than rest:

"If the message hadn't been [to expect to recover in] around two weeks, l'd have been more cautious at first, ... the doctor I saw in A/E described Covid as the gift that keeps on giving and at four weeks I thought that felt like a long time, and now five months on it feels like a very long time" Sarah

There was also an absence of information about the fluctuating nature of symptoms. People expected the abatement of one symptom was the 'end' of their Covid19 experience and were devastated when a new symptom appeared. The focus group believed lack of current information influenced the way in which some healthcare professionals responded, with some people being told that after four weeks that they should 'just get on with it'. NHS 111 advice was said to be basic: 'stay at home and take paracetamol unless you are ill enough to go to hospital'. One person reported ringing 111 to say they hadn't recovered yet and was told 'well you should have'. The mismatch between what people were being told and what they were experiencing created anxiety about why they hadn't recovered.

All the focus group members felt it had been hard to learn to lower their expectations of recovery but that gaining realistic goals and pacing themselves was important in moving forward. They recognised that evidence is only just emerging and it is hard to give definitive advice but felt official channels and health professionals should be less rigid in their description of symptoms and in their advice about recovery journeys.

\section{Symptom journey}

\section{Key points}

There is a lack of understanding that people living with Covid19 can suffer from a wide range of interconnected symptoms, and that even if not individually severe they can collectively leave people severely debilitated. This means many are relying on peer support through social media channels

Our focus group reported that symptoms did not follow a linear journey as official channels implied. Rather than move from an 'acute' phase to either a chronic or a recovery phase, they talked of symptoms moving round their body. They described multiple symptoms across respiratory, cardiovascular, urological, neurological/cognitive, dermatological, gastrointestinal, and musculoskeletal systems. Their Covid19 experience didn't always start with cough, fever and breathlessness; for some, symptoms such as cough came later on rather at the beginning. For others, atypical symptoms including tachycardia and fainting were the first sign of infection. 
Other severe symptoms occurred some weeks into their illness. These included high blood pressure and unbearable 'heart pain':

"Definitely there was so much changing over time, it's what makes it so stressful, you're going along and feeling that maybe some symptoms are behind you, but then you come up with some other completely different thing, I think if it were not so frightening it would be laughable. I first had symptoms on $5^{\text {th }}$ March but it wasn't until mid-May my heart symptoms came on and they were the most frightening of all" Patricia

The biggest challenge for many was not the individual symptoms, but their unpredictability and the dashed hopes of recovery after a few good days:

"It's a roller coaster rather than an incline, I'm going up and down and up and down. If I do too much I feel worse, and if I don't do enough I feel worse and so when I feel better I have to bank the energy" Nigel

Group members described a wide range of symptoms across all body systems. They felt strongly that the symptoms were causing their anxiety and not (as some health professionals have suggested to them) the other way around. The uncertainty about the cause and restricted access to diagnostics meant these people were left isolated, wondering whether they had a concurrent disease that was being missed. They felt that better information about the range of people's experiences would give them some reassurance as well as sharing details of coping strategies.

\section{Being doubted}

\section{Key points}

A lack of recognition of the pattern of symptoms by the public and by healthcare services meant people frequently felt their experiences were not believed and this left many feeling isolated and alone in coping with their disease.

The widespread expectation that Covid19 has an acute and a recovery phase was thought by all of the group to contribute to a perception of their stories of a different journey not being believed, either by health professionals, by employers, or indeed by friends and family. This lack of acknowledgement made people feel very isolated and query themselves:

"None of us knew this [the symptoms] because we're all on our own, in a little bubble, thinking I'm the only one. Why am I the one who has still got it?" Julia 


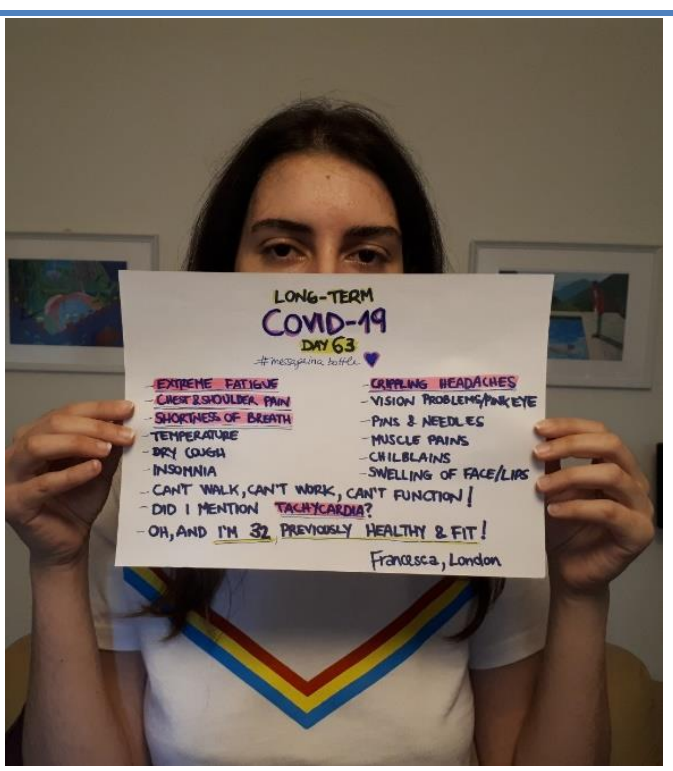

"My journey with COVID-19 began on 27th April. I'm still unwell five months on and haven't been able to resume a normal life since. My worst and scariest experience with this illness was in week 6, when I was rushed to A\&E as I had a sudden relapse of symptoms and found myself gasping for air, with the top of my head numb and tingling and a headache so blinding that I couldn't keep my eyes open. I got worse in the hospital and was shaking visibly, so much so that the nurse couldn't perform an ECG as I just couldn't stay still.

Despite having been diagnosed with suspected Covid by my GP and a doctor in a Covid clinic (swab testing wasn't available to the public at the time) and told I had pleurisy during a visit to A\&E two weeks earlier, the doctor on duty didn't take this into account. Instead, he dismissed me with anxiety, advising a course of anti-depressants, and chose not to investigate these concerning symptoms further. Of course I was anxious, but that was a consequence of the physical symptoms, not the cause!

I would later learn from a neurologist that what I experienced on that day were clear neurological symptoms that should have been investigated promptly. To be brushed off like this when so little was known at the time of the damage Covid can cause was disheartening and very upsetting." Francesca

The narrow and unchanging definition of Covid19 symptoms by health professionals and advice lines was seen to reinforce some health professionals' scepticism about atypical journeys. On the one hand, some members of the group reported that if they had tested positive, every symptom (however potentially serious) was attributed to Covid19 without the need for further investigation. People described having done their own research into consequences of Covid19 and asking for blood tests and X-rays but being told they weren't needed. On the other hand, others reported multiple investigations for other diseases rather than being offered support and therapy for what they believed to be long term effects of Covid19. Both approaches demanded both emotional and physical energy that people didn't have in order to get support appropriate to their individual need. The cyclic nature of the ongoing effects was seen as a major contributor for the scepticism: 
"It's a crafty illness, and it's a doubting illness because, it makes you doubt yourself and it makes people doubt you. My symptoms started in February but when you think you're over it then you start to get a bitter taste, then a few days later the fatigue comes back" Lee

This cyclical nature was seen to be very difficult for family and friends who try to be positive and celebrate any small improvements and find a 'relapse' hard to cope with.

The focus group expressed the relief of finding a healthcare professional who simply listened to them and acknowledged their symptoms.

"I finally found a GP who took me seriously last Saturday when I was at the point of crying talking to her, just understanding that people's symptoms are real and diverse." Andres

\section{Support}

Key points

Lack of diagnosis makes accessing services difficult. Primary care, community and helpline staff need better training and knowledge about the ongoing effects of Covid19

Whilst some of the group felt that all attention was directed towards people who had been admitted to hospital, two of the group who had been treated in critical care described how the follow up had not included their 'Long Covid' symptoms and focused primarily on respiratory function. Both received post intensive care discharge support but once that stopped they were left feeling as uncertain and as unsupported as those who had never been admitted to hospital. There appeared to be no recognition that ongoing Covid19 may be different to Post-intensive care syndrome (PICS) or that some people may experience both.

The focus group also reported that the need for ongoing support for family members:

"My wife, who is the one who has been dealing with this more than I have, has felt a number of times that she's been left on her own and not that many questions have been answered' Nigel

Finding the right support for multiple symptoms has been challenging. There was concern that health services, even at a specialist level, are based on single system symptoms:

"There's no point dealing with our symptoms one at a time when they are so varied and also interlinked. I'd like one person who could look at all of it. Heart rate, fatigue etc. I'm getting my health advice from the [Facebook] group but it would be nice to have some medical input!" Sarah

There was some support for the idea of one stop Covid clinics:

"What would be most helpful is if all main hospitals could have a Covid clinic that had experts from respiratory, cardiology, rheumatology, neurology, physiotherapy etc, so you could go along for half a day and see people from these different departments, 
they can refer you for tests and you can get a plan in place, We are having such a range of symptoms that GPs are struggling to know what to do with you" Nikki

Alternatively there was a view that it would be helpful if people living with Covid19 could have a 'quarter back' or case manager to oversee and coordinate investigations and support services across different medical specialities.

The group described challenges with accessing primary care. Many had only had telephone contact and even those who had had video consultations found that they were inadequate for conveying the nature of their symptoms. One member of the group was ill for over five months and paid to see a GP privately in order to see someone face to face and to have her chest listened to.

Members of the group recognised that GPs are also frustrated by this but noted a risk averse attitude in health services that was also restricting access:

"Most Covid patients are no longer infectious after 2- 3 weeks, but many of us are refused access to healthcare if we still have symptoms, which may last for months" Nora

There was a strong feeling that healthcare professionals, from 111 to GPs, needed more detailed guidance, including standardised criteria for seeking further investigations and referrals to specialist services. The group felt that this would be helped by having a working diagnosis:

"I never had a positive test as was tested eight weeks after symptoms started and also, three of the referrals my GP made (two respiratory and one neurology) were refused by two different hospitals on the grounds that a) they only checked Covid confirmed patients $b)$ that they needed extra tests which weren't done on me at A\&E" Francesca

It was strongly felt that diagnosis should be based on national guidelines. This should include history of symptoms and clinical observation and that a lack of laboratory tests should not be an exclusion to a working diagnosis. A confirmatory diagnosis would be psychologically helpful to validate their experience and to reduce fear of unknown alternative diagnoses. Whilst a precise definition is not yet possible, logging the symptoms now may help both individuals and researchers in the future:

"My nurse practitioner said, you're not crazy, it probably is connected, let's keep testing until we find something or we don't but at least we have it all on the record that you've had these issues since you got Covid, and you never had them before" Kelly

Focus group members understood that there were no magic cures, but were looking for practical advice on coping strategies that go beyond basic advice. This meant detail on what is known about living with Covid19 symptoms and treatments as well as describing what the uncertainties are:

"National websites largely unhelpful in advice, didn't add to what was already known, e.g. breathlessness" Penny

The British Lung Foundation and Asthma UK's joint website www.post-covid.org.uk was cited as providing high quality information on symptom management. Advice on 
the range of symptoms and duration was also needed by employers who are unclear what to expect of those with ongoing effects. The UK Sepsis Trust was commended for their information for people who had been in critical care on returning to work.

In conclusion, the focus group feel there needs to be more education for primary healthcare providers to ensure that potential suffers are listened to and understood, all of their symptoms assessed, appropriate tests/investigations arranged and care plans agreed.

The focus group described a very wide range of symptoms. These are echoed in this video from the LongCovidSoS group.

https://www.youtube.com/watch?v=IleOoS A4c8

\section{https://www.longcovidsos.org/}

Have you been living with Covid19? Does your experience match that of our focus group? We'd like to understand more and invite you to fill in this survey

\section{Services needed}

Both people who have been discharged from hospital and those never admitted need health and social care support and rehabilitation as there is currently no 'cure'. As with all long term conditions, this needs to go beyond the person with the symptoms to the family and carers and in a range of settings, including people's own homes and nonhospital residential settings. Consideration of how to provide this and the skills required to do so is an urgent priority and should include independent, third sector and voluntary providers who are already providing social support. This extends beyond healthcare and research questions need to include social impact (including the impact of moral judgements/stigma of having 'Long Covid') and returning to employment.

Our steering group debated whether this support needs to be a specialist service or part of all primary and community care services. Comparisons were drawn with the emergence of HIV in the 1980s and the arguments for and against specialist services. The contribution of different professions and their education about living with Covid19 also needs to be studied to determine the optimal service and workforce configuration.

The changing and multiple symptoms of ongoing Covid19 are not well served by the way healthcare is currently organised. Covid19 is a multi-system disease and people's experience echoes the challenges already experienced by people with multiple comorbidities, who experience limited service integration. As an example, one member of the steering group described a woman admitted to hospital for respiratory support who needed psychological support. Because of the way services are provided, mental health teams were unhappy about providing PTSD support in hospital and said she could self-refer once she had been discharged home.

The support needs of people who have been ill for long periods with any disease have been recognised for some time. One study found $64 \%$ of people who were treated in intensive care had problems with cognitive impairment, disability, or depression three 
months after discharge, and $56 \%$ still had problems at 12 months ${ }^{17}$. Early in the pandemic there was an expectation that people who had been critically ill with Covid19 and had extended stays in intensive care would need intensive rehabilitation. It is also apparent that they may have suffered long term disabilities related to other events triggered by the seriousness of their illness (e.g. stroke, or loss of muscle tone) rather than by the virus itself, and may therefore require specialised rehabilitation services related to the acuity of their illness rather than Covid19 specifically.

Whilst post hospital care is important, the British Society of Rehabilitation Medicine notes there are people who were never admitted to hospital but who still have ongoing needs for rehabilitation support after recovering from Covid, or Covid-like symptoms. Indeed, we heard from people in our focus group who had been ventilated in critical care and received post discharge rehabilitation care who said their ongoing needs are the same as those who had not received any hospital care, suggesting that care models need to be tailored specifically to Covid19.

\section{Resources}

\section{Health organisation and policy research resource centres}

Much of the research undertaken to date has understandably been biomedical, but attention is now moving to organisational and policy issues. The International Longterm Care Policy Network (ILPN) and Care Policy and Evaluation Centre (CPEC) at the London School of Economics and Political Science (LSE) have established a website to curate the impact of Covid19 on people who rely on long-term care (including unpaid care) and those who provide it. It aims to share information about policy and practice measures to mitigate the impact of Covid19 in long-term care to analyse the long-term implications of Covid19 for long-term care policy. The website includes research reports and guidance relating to long-term care from across the world https://ltccovid.org/

The Covid19 Evidence Network to support Decision-making (COVID-END) at McMaster University supports decision makers to coordinate the evidence syntheses, technology assessment and guidelines being produced. This includes a living review of evidence on health systems and a living review of clinical management of Covid19 and pandemic-related health issues. This site tracks other living reviews, such as the Living Systematic Review of Mental Health in Covid19 being conducted by DEPRESSD, an international collaborative project involving experts in health policy, psychiatry and statistics. https://www.mcmasterforum.org/networks/covid-end

The Health Foundation has been analysing government policy and is running a live Covid19 policy tracker of national policy and health system responses to Covid19 in England.

https://www.health.org.uk/news-and-comment/charts-and-infographics/covid-19policy-tracker

They have also launched a programme funding applied research studies that seek to understand how health and social care service delivery has changed in light of 
Covid19 and the impact of Covid19 on health inequalities and the wider determinants of health. https://www.health.org.uk/funding-and-partnerships/programmes/covid-19research-programme

\section{Expert consensus support resources for service providers}

There are many websites providing expert consensus advice and emerging evidence. We provide links to a small selection here. Some are focused on a particular symptom and others on the broad experience. We have grouped the resources around particular themes, but many sites have useful information beyond these categories. We have divided them into sites for healthcare providers and sites for people with symptoms but there are similar overlaps.

\section{Advice for healthcare providers}

\section{Rehabilitation}

The Stanford Hall consensus statement for post Covid19 rehabilitation, drawn up by experts from a broad range of rehabilitation disciplines underlines the fact that Covid19 is a multisystem disease. A review of evidence around rehabilitation following previous coronavirus infections resulted in 32 evidence-based recommendations for post Covid19 rehabilitation.

https://bjsm.bmj.com/content/54/16/949.abstract

The British Society of Rehabilitation Medicine has issued a working document that sets out their recommendations for rehabilitation services for adults in the wake of the Covid-19 pandemic - in particular, the role of specialist rehabilitation to support patients with more complex rehabilitation needs following hospitalisation. https://www.bsrm.org.uk/downloads/covid-19bsrmissue2-11-5-2020-forweb11-5-

\section{0.pdf}

The British Thoracic Society recommend a programme on the management of fatigue, breathlessness, and mood disturbances (including symptoms of anxiety and depression). They recommend that people who received respiratory support in hospital should have a clinical review to detect issues at 4- 6 weeks post-discharge (this may be remote where feasible) and a face to face review at 12 weeks postdischarge. https://www.brit-thoracic.org.uk/about-us/covid-19-information-for-therespiratory-community/

The National Institute for Health and Care Excellence (NICE) has guidance on 'Rehabilitation after critical illness in adults [CG83]' Published March 2009' https://www.nice.org.uk/guidance/cg83

The Faculty of Intensive Care Medicine published provisional guidance 'Recovery and Rehabilitation for Patients Following the Pandemic' in May 2020. This suggests that people with Covid19 are likely to need significant follow up, which general practice is unlikely to be able to provide. It discusses a number of models of service provision to meet the ongoing needs.

www.ficm.ac.uk/sites/default/files/ficm rehab provisional guidance.pdf 
The Chartered Society of Physiotherapy has developed Covid19 rehabilitation standards for the delivery of physiotherapy within a multidisciplinary care context.

https://www.csp.org.uk/news/coronavirus/clinical-guidance/rehabilitation-

coronavirus/covid-19-rehabilitation-standards

\section{Primary Care}

Guidance for General Practitioners on managing patients living with Covid19 was published in the BMJ. This emphasises the need to make a diagnosis without confirmed laboratory tests and to consider the breadth of symptoms, not just single systems. https://www.bmj.com/content/370/bmj.m3026

\section{Fatigue}

Physios for ME (Myalgic Encephalomyelitis) have produced guidance on the potential complication for Post-Viral Fatigue Syndrome (PVFS).

https://www.physiosforme.com/covid-19

The (Myalgic Encephalomyelitis) ME association has produced a leaflet on Post-viral fatigue (PVF) and Post-Viral Fatigue syndrome (PVFS) following coronavirus infection

https://meassociation.org.uk/wp-content/uploads/MEA-PVF-and-PVFS-Following-

Coronavirus-Infection-30.04.20.pdf

\section{Mental health}

A consistent theme that emerges is the way Covid19 can challenge people's mental health, ranging from mild anxiety to post traumatic stress disorder. The British Psychological Society has established a Covid19 Coordinating Group and their website has details of work streams and advice for the general public https://www.bps.org.uk/coronavirus-resources

The Covid Trauma Response Working Group, a collaboration of psychological trauma specialists, wellbeing leads at NHS Trusts and people with lived experience of psychological trauma have produced guidance on screening and active monitoring for post-traumatic stress disorder (PTSD) and other mental health consequences in people recovering from severe Covid19 illness. https://232fe0d6-f8f4-43eb-bc5d6aa50ee47dc5.filesusr.com/ugd/6b474f 733283bf71ce4295b915dffa86886280.pdf

The Oxford Centre for Anxiety Disorders and Trauma (OxCADAT) have resources for mental health professionals working with PTSD post Covid19 which may be relevant.www.oxcadatresources.com

\section{Living with Covid19 and other Long-term Conditions}

The National Institute for Health and Care Excellence (NICE) has produced several rapid guidelines for managing conditions alongside Covid19 including:

NICE (2020a) COVID-19 rapid guideline: severe asthma. National Institute for Health and Care Excellence. https://www.nice.org.uk/guidance/ng166,

NICE (2020c) COVID-19 rapid guideline: acute myocardial injury. National Institute for Health and Care Excellence. https://www.nice.org.uk/guidance/ng171 
NICE (2020e) COVID-19 rapid guideline: community-based care of patients with chronic obstructive pulmonary disease (COPD). https://www.nice.org.uk/guidance/ng168

Using digital technologies

A lot of attention has focused on using digital platforms to provide care whilst social distancing. This also has potential for people living with Covid19 who may find visiting clinics fatiguing.

The Royal College of Psychiatrists has published a guide to providing mental health care via remote consultation. https://www.rcpsych.ac.uk/mental-health/treatmentsand-wellbeing/remote-consultations-and-covid-19

The General Medical Council has issued guidance for doctors on remote consultations https://www.gmc-uk.org/ethical-guidance/ethical-hub/remoteconsultations

The Chartered Society of Physiotherapy has published COVID-19: guide for rapid implementation of remote consultations. https://www.csp.org.uk/publications/covid19-guide-rapid-implementation-remote-consultations

The University of Oxford Nuffield Department of Primary Care Health Sciences has also published guidance on video consultations.

https://www.phc.ox.ac.uk/research/resources/video-consulting-in-the-nhs

The NIHR Policy Research Unit, Older People and Frailty, has produced a briefing paper on using digital technologies to deliver strength and balance exercises for falls prevention amongst older people. https://www.opfpru.nihr.ac.uk/covid-19research/rr7-covid-19-technology-for-strength-and-balance/

\section{Workforce reconfigurations}

Most care for people living with ongoing effects of Covid19 is going to be provided in the community. This will necessitate an expansion of the workforce. The Queen's Nursing Institute has developed competencies for nurses in General Practice transitioning to Community Nursing during the Covid19 pandemic.

https://www.qni.org.uk/resources/minimum-bridging-competencies-for-general-

practice-nurses-transitioning-to-community-nursing

\section{Advice for people living with Covid19}

Recovery

NHS England has published a website that provides people with advice on managing the ongoing effects of and recovery from Covid19. The website includes advice on eating well, sleeping well, getting moving again, help for friends, family and carers on understanding what the person has been through and coping with bereavement and 
grief. People who need more support can be referred by a healthcare professional to a programme called 'Your COVID Recovery'. Patients can have a face-to-face consultation with their local rehabilitation team of physiotherapists, nurses and mental health specialists and may be offered a personalised package of online-based aftercare lasting up to 12 weeks, including an online peer-support community, exercise tutorials that people can do from home and online mental health support from a psychologist. https://www.yourcovidrecovery.nhs.uk/

Homerton Hospital NHS Foundation Trust produced an information pack with practical tools and tips for self-management of symptoms. https://www.hackneycitizen.co.uk/wp-content/uploads/Post-COVID-19-informationpack-5.pdf

The Chartered Society of Physiotherapy has created a suite of resources, including videos, to help people manage their symptoms. https://www.csp.org.uk/publicpatient/covid-19-road-recovery

Post critical care recovery

The UK Sepsis Trust has developed guidance on Recovery after Critical IIIness and Returning to work Following Critical Illness https://sepsistrust.org/covid-19-recoveryresponse/

ICUsteps, a charity run by former intensive care patients and relatives to improve the care and support available to patients recovering from critical illness during their long recovery has a number of resources specifically related to Covid19

https://icusteps.org/home/covid-19

\section{Breathing problems}

British Lung Foundation and Asthma UK have developed a website for people with post-Covid breathing problems. It has separate advice sections for people who stayed at home, who were looked after in hospital and who were looked after in intensive care. It has detailed practical advice for self-care and indications of when to seek professional help. www.post-covid.org.uk

Mental Health

The COVID Trauma Response Working Group host a comprehensive guide for the public on PTSD following critical illness and intensive care. https://www.traumagroup.org/

Psychology Tools website had a number of guides, including 'Patient Guide -Critical Illness, Intensive Care, and Post-Traumatic Stress Disorder' https://232fe0d6-f8f4$43 \mathrm{eb}-\mathrm{bc} 5 \mathrm{~d}-$

6aa50ee47dc5.filesusr.com/ugd/c5f932 eb188663c2634f9ea8d9247909f2c3b0.pdf

\section{Fatigue}

The Royal College of Occupational Therapists has produced guidance for patients on how to manage post-viral fatigue after COVID-19 
https://www.rcot.co.uk/node/3540 with specific advice on conserving energy whilst undertaking activities of daily living https://www.rcot.co.uk/conserving-energy

Please share any resources that cover other areas. We are especially interested in resources for children and young people, people with learning disabilities, older people and homeless people. Please let us know of any information here

\section{Commissioned research}

The National Institute for Health Research responded rapidly to the outbreak of Covid19 and commissioned urgent biomedical and public health research - research that needed to take place during the emergency phase of the pandemic and that will deliver an impact within 12 months. It is now investing in a rolling programme of 'recovery and learning' research. It has also launched a call for global health research to tackle COVID-19 in low- and middle-income countries, in partnership with UK Research and Innovation. For more details of NIHR funding plans, please visit https://www.nihr.ac.uk/covid-19/.

We have mapped a selection of current research listed on NIHR databases and the NHS Health Research Authority Covid19 database. We recognise that this is not a comprehensive search and welcome feedback on important studies that we have not listed. We have mapped the studies against themes identified by our focus group of people living with Covid19

\section{Understanding the continuing effects of Covid19}

University College London is leading the UK arm of an International Observational Study of Outpatients with SARS-CoV-2 Infection. This is an observational cohort study of adults who have been assessed as not requiring hospitalization to estimate the rate of disease progression for adults who seek testing and test positive for SARS-CoV-2. https://www.ucl.ac.uk/global-health/research/z-research/international-sars-cov-2infection-observational-study-icos

Queen Mary University of London is leading the COVIDENCE Study, a national initiative which collects data from volunteers through baseline and monthly follow-up on-line questionnaires. It aims to advance understanding of risk factors for coronavirus disease among UK adults, exploring how quickly people recover and whether there are any long-term complications of this illness. https://www.qmul.ac.uk/covidence/about-the-covidence-uk-study/

The NIHR funded Post Hospitalisation COVID19 study (PHOSP-COVID) will follow 10,000 patients who have been hospitalised for one year to gain a comprehensive picture of the impact of Covdi19 on longer term health outcomes. https://www.phosp.org/ 
The NIHR together with the British Heart Foundation is running the COVID-HEART study which will investigate how often, and in what way, the heart becomes damaged, and how it recovers 6 months later. https://www.bhf.org.uk/forprofessionals/information-for-researchers/national-flagship-projects

The NIHR University College Hospitals Biomedical Research Centre (BRC) and the British Heart Foundation are leading the UK arm of European-wide CAPACITY-COVID project, will seeks to explore how cardiovascular disease and risk factors increase the risk of developing severe complications of Covid19, and which acute cardiovascular complications are most common in patients who develop Covid19.

https://www.bhf.org.uk/for-professionals/information-for-researchers/nationalflagship-projects

\section{Managing symptoms, rehabilitation and recovery}

The Defence and National Rehabilitation Centre (DNRC) M-COVID study is investigating the complications and outcomes of Covid19 infection in a young/middle aged physically active population (in this case a military population) who have had a range of mild to severe initial infection. This demographic provide the majority of the UK workforce especially in physically active/manual jobs, but to date most studies are focussing on elderly patients with severe acute disease. There are potentially many younger patients who have had mild/moderate acute Covid19 who were not admitted to hospital but who have on going functional, and in particular, exercise limitations. The study includes assessment of heart and lung function. Functional, mental health, cognitive and neurological outcomes will also be investigated. Participants will be followed up for 12 months after baseline assessment. The primary outcomes are cardiopulmonary function as assessed by Cardiopulmonary Exercise Testing (CPET) and the 6 minute walk test at 6 months.

Brighton University is testing whether critically ill patients who have been on a ventilator can benefit from remote prescribed exercise following their discharge from hospital. One hundred patients will be given remote exercise rehabilitation plans, advice and will be given a fitness watch to track their physical activity. Their physical health will be monitored by follow-up appointments three months, six month and one year after being discharged from hospital.

https://www.brighton.ac.uk/news/2020/monitoring-recovery-from-covid-19

\section{Providing services to support people living with Covid 19}

The NIHR Mental Health Policy Research Unit is leading a mixed methods investigation of impact on mental health services of the Covid19 impact and ways of mitigating it (MH PRU) https://www.ucl.ac.uk/psychiatry/covid-19-project 
The NIHR School for Primary Care Research is undertaking rapid research into how primary care has managed demand and implemented alternatives to the face-to-face consultation. https://www.spcr.nihr.ac.uk/projects/Rapid-COVID-19-intelligence-toimprove-primary-care-response-\%28RAPCl\%29-project

\section{Working digitally}

Oxford University is evaluating the scaling up of 'Remote-by-Default' models of care. There will be two phases, a rapid evaluation phase that maps different models and longitudinal comparative case studies of primary care sites across England and Scotland, generating rich in-depth narratives of scale-up of 'remote-by-default' models in primary care and the impacts on staff and patients. https://www.phc.ox.ac.uk/covid19/projects/remote-by-default-care

\section{Early conclusions}

The first phase of research has focused on public health measures. Management of acute disease and attention is starting turn to the ongoing effects of Covid19. The evidence we have drawn together demonstrates a significant and growing burden of need in a significant proportion of people who have are living with ongoing Covid19.

We found that

- There is a current widespread perception that people either die, get admitted to hospital or recover after two weeks but it is increasingly clear that for some people there is a distinct pathway of ongoing effects.

- A working diagnosis that is recognised by healthcare services, employers and government agencies would facilitate access to much needed support and provide the basis for planning appropriate services. While it is too early to give a precise definition, guidance on reaching a working diagnosis and a code for clinical datasets is needed

- People experience a wide range of fluctuating and multi-system symptoms that need to be acknowledged. A common theme is that symptoms arise in one physiological system then abate only for symptoms to arise in a different system.

- Ongoing Covid19 needs to be considered holistically (both in service provision and in research).

- The varying degrees of dependency mean support in the community should be considered alongside hospital one stop clinics.

- There are significant psychological and social impacts that will have long term consequences for individuals and for society if not well managed.

- Health and social care services are not equipped to support people living with Covid19. Staff need better information and education on the ongoing effects.

- There is an urgent need to better understand the symptom journey and provide realistic expectations about progression. 
But many questions remain unanswered

- What are the risk factors and therefore who is at greatest risk of experiencing long term problems?

- What is the impact of living with Covid19 on families and carers? How can social support, including that provided by voluntary agencies, help?

- What are the financial pressures on previously economically active people?

- Acute Covid19 infection has already had a disproportionate effect on certain parts of the population. Is this mirrored in ongoing Covid19?

Next steps

We will update this review as and when the findings of the studies identified above are published. We will continue to scan the NIHR funding programme for new research studies. We are also hoping that readers of this site will help to us to crowd source other resources.

We will be running a series of online events to encourage discussion, staring with webinars in November 2020 and we will be publishing the proceedings. Please click here to learn more and to book your place

Please share any thoughts on this review with us here

\section{References}

1 Carfì, A., Bernabei, R. and Landi, F. (2020) Persistent symptoms in patients after acute COVID-19. JAMA 324(6):603-605

https://jamanetwork.com/journals/jama/fullarticle/2768351

2 NHS England (2020) After-care needs of inpatients recovering from COVID-19 https://www.england.nhs.uk/coronavirus/publication/after-care-needs-of-inpatientsrecovering-from-covid-19/

3 Murray, A., Gerada, C., Greenhalgh, T. (2020) We need a Nightingale model for rehab after covid-19 HSJ April 8 https://www.hsj.co.uk/commissioning/we-need-anightingale-model-for-rehab-after-covid-19-/7027335.article

4 Public Health England (2020) COVID-19: long-term health effects https://www.gov.uk/government/publications/covid-19-long-term-health-effects/covid19-long-term-health-effects

5 Patient-Led Research Team (2020) What Does COVID-19 Recovery Actually Look Like? An Analysis of the Prolonged COVID-19 Symptoms Survey https://patientresearchcovid19.com

6 COVID Symptom Study https://covid.joinzoe.com/post/covid-long-term 
7 Royal College of General Practitioners (2020) General practice in the post Covid world Challenges and opportunities for general practice https://www.rcgp.org.uk//media/Files/News/2020/general-practice-post-covid-rcgp.ashx?la=en

8 Wade, D.T. (2020) Rehabilitation after COVID-19: an evidence-based approach. Clinical Medicine Vol 20, No 4

917 NICE Coronavirus - COVID 19 Scenario: Management of other medical conditions https://cks.nice.org.uk/coronavirus-covid-19\#!scenario:1 accessed August 2020

10 Hui DS, Joynt GM, Wong KT, et al. (2005) Impact of severe acute respiratory syndrome (SARS) on pulmonary function, functional capacity and quality of life in a cohort of survivors Thorax 60(5):401-409.

11 Das KM et al. Follow up chest radiographic imaging in patients with MERS-CoV after recovery. Indian J Radiol Imaging 2017;27(3)342-349

12 Dasgupta, A., Kalha, A. and Kalra, S. (2020) Long term complications and rehabilitation of COVID-19 patients. The Journal of the Pakistan Medical Association, 70(5), pp.S131-S135.

13 Chan, K.S., Zheng, J.P., Mok, Y.W., Li, Y.M., Liu, Y.N., Chu, C.M. and Ip, M.S. (2003) SARS: prognosis, outcome and sequelae. Respirology, 8, pp.S36-S40.

14 Tansey, C.M., Louie, M., Loeb, M., Gold, W.L., Muller, M.P., de Jager, J., Cameron, J.I., Tomlinson, G., Mazzulli, T., Walmsley, S.L. and Rachlis, A.R. (2007) One-year outcomes and health care utilization in survivors of severe acute respiratory syndrome. Archives of internal medicine, 167(12), pp.1312-1320

15 Gardner, P.J. and Moallef, P. (2015) Psychological impact on SARS survivors: Critical review of the English language literature Canadian Psychology 56(1), p.1235

16 Gordon, A., Goodman, C. Achterberg, W., Barker, R., Burns, E., Hanratty, B., Martin, F., Meyer, J., O'Neill, D.,Schols, J., Spilsbury, K. (2020) Commentary: COVID in Care Homes-Challenges and Dilemmas in Healthcare Delivery Age and Ageing, 49:5. 701-705 https://doi.org/10.1093/ageing/afaa113

17 Marra, A., Pandharipande, P.P., Girard, T.D., Patel, M.B., Hughes, C.G., Jackson, J.C., Thompson, J.L., Chandrasekhar, R., Ely, E.W. and Brummel, N.E. (2018) Co-occurrence of post-intensive care syndrome problems among 406 survivors of critical illness Critical Care Medicine 46(9), p.1393.

\section{Steering Group}


Group Captain Alex Bennett Defence Medical Rehabilitation Centre / Loughborough University Professor of Rheumatology and Rehabilitation Medicine

Squadron Leader Rob Barker-Davies Ministry of Defence Rehabilitation Medicine

Professor Roshan das Nair University of Nottingham Professor of Clinical Psychology and Neuropsychology

Caroline Morrice Guillain Barre and Associated Inflammatory Neuropathies (GAIN) CEO

Dr Hannah Murray

Oxford Centre for Anxiety Disorders and Trauma Research Clinical Psychologist

Dr David Murphy

Dr David Oliver British Psychological Society President Geriatrician

Dr Philip Pearson Royal Berkshire NHS FT

Northampton General Hospital NHS Trust Respiratory Physician

Professor Sarah Tyson

University of Manchester Professor of

Rehabilitation

Tara Lamont

Advisor

Liz Jones

Professor Julienne Meyer

Dr Crystal Oldman

Dr Vanessa Abrahamson

University of Southampton Senior Scientific

National Care Forum

Policy Director

National Care Forum

R\&D Advisor

Queen's Nursing Institute Chief Executive

University of Kent

Research Associate

\section{Patient Reference Group}

Kelly Keeney

Lee David Bowen

Nigel Simm

Penny Tassoni MBE

Edward Jarvis

Identities known to NIHR but not disclosed

Nikki

Francesca

Andres

Jo

Pretty

Nora

Sarah

Julia

Patricia 